\title{
On Goursat-Darboux boundary-value problem for systems of nonlinear-differential equations of hyperbolic type
}

\author{
V. V. Marynets and K. V. Marynets
}




\title{
ON GOURSAT-DARBOUX BOUNDARY-VALUE PROBLEM FOR SYSTEMS OF NON-LINEAR DIFFERENTIAL EQUATIONS OF HYPERBOLIC TYPE
}

\author{
V. V. MARYNETS AND K. V. MARYNETS
}

Received 3 September, 2013

\begin{abstract}
We construct the modification of the two-sided method with improved characteristics of convergence for approximate integration of boundary-value problem for systems of secondorder non-linear differential equations of the hyperbolic type on the plane when the bound of the domain of the independent variables consists of the pair of characteristics.
\end{abstract}

2010 Mathematics Subject Classification: 34B10; 34B15

Keywords: partial differential equations, Goursat-Darboux boundary-value problem, two-sided method, approximations

\section{INTRODUCTION}

In the theory of partial differential equations of the hyperbolic type the basic classical problems are already deeply studied. The domain of the independent variable is bounded by two pairs of parallel lines or by two pairs of characteristics, the pair of characteristics crossing "free" curve, etc $[1,2,4]$.

Boundary-value problems for equations of hyperbolic type in the case of more complicated structure of the bound of the domain are studied less frequently.

In this work we generalize results obtained before in [6] and construct the modification of the two-sided method for approximate integration of the boundary-value problem for systems of second-order non-linear differential equations of hyperbolic type on the plane, when the bound of the domain consists of the pair of characteristics of the given system.

Note that constructive modifications of the numerical-analytic algorithm of investigation of the boundary-value problems for non-linear systems of ordinary differential equations subjected to different types of boundary restrictions have been studied in detail in $[3,7,8]$.

Let us observe that the domain

$$
D=\bigcup_{s} D_{s} \subset \mathbb{R}^{2}, s=1,2,3
$$


where

$$
\begin{gathered}
D_{1}=\left\{(x, y) \mid x \in\left(x_{0}, x_{1}\right], y \in\left(y_{0}, y_{1}\right]\right\}, \\
D_{2}=\left\{(x, y) \mid x \in\left[x_{0}, x_{1}\right], y \in\left(y_{1}, g_{1}(x)\right)\right\}, \\
D_{3}=\left\{(x, y) \mid x \in\left(x_{1}, x_{2}\right], y \in\left(g_{2}(x), y_{1}\right]\right\},
\end{gathered}
$$

$x_{0}<x_{1}<x_{2}, y_{0}<y_{1}<y_{2}, y=g_{r}(x)\left(x=k_{r}(y)\right), x \in\left[x_{r-1}, x_{r}\right], r=1,2$ are "free" curves [4], and $g_{1}\left(x_{r-1}\right)=y_{r}, g_{2}\left(x_{r}\right)=y_{r-1}, g_{r}^{\prime}(x)>0$.

The problem is to find the solution of the system of differential equations

$$
L_{2} U(x, y)=f(x, y, U(x, y)):=f[U(x, y)],
$$

subjected to boundary restrictions of the form:

$$
\begin{gathered}
U\left(x_{0}, y\right)=\Psi(y), U\left(x, y_{0}\right)=\Phi(x),(x, y) \in \bar{D}_{1}, \\
\Psi(y) \in C^{1}\left[y_{0}, y_{1}\right], \Phi(x) \in C^{1}\left[x_{0}, x_{1}\right], \Psi\left(y_{0}\right)=\Phi\left(x_{0}\right), \\
U\left(x, g_{r}(x)\right)=\Omega_{r}(x), x \in\left[x_{r-1}, x_{r}\right], \Omega_{r}(x) \in C^{1}\left[x_{r-1}, x_{r}\right], r=1,2, \\
\Omega_{1}\left(x_{0}\right)=\Psi\left(y_{1}\right), \Omega_{2}\left(x_{1}\right)=\Phi\left(x_{1}\right),
\end{gathered}
$$

in the space of vector-functions $C^{*}(\bar{D}):=C^{(1.1)}(D) \cap C(\bar{D})$, where

$$
L_{2} U(x, y):=U_{x y}(x, y)+A_{1}(x, y) U_{x}(x, y)+A_{2}(x, y) U_{y}(x, y),
$$

$U(x, y):=\left(u_{i}(x, y)\right), f[U(x, y)]:=\left(f_{i}[U(x, y)]\right), i=\overline{1, n} \Omega_{r}(x):=\left(\omega_{i, r}(x)\right)$, $\Psi(y):=\left(\psi_{i}(y)\right), \quad \Phi(x):=\left(\phi_{i}(x)\right) \quad$ are some given vector-functions, $A_{r}(x, y):=\left(\delta_{i, j} a_{i, j}^{(r)}\right), r=1,2, j=\overline{1, n}$ are given matrixes and $\delta_{i, j}$ is the Kronecker symbol.

Function $U(x, y)=U_{s}(x, y),(x, y) \in \bar{D}_{s}, s=1,2,3$ is the solution of the boundaryvalue problem (1.1)-(1.3), where $U_{1}(x, y),(x, y) \in \bar{D}_{1}$ is the solution of the Goursat problem (1.1), (1.2), $U_{s}(x, y), s=2,3,(x, y) \in \bar{D}_{s}$, are the solutions of the Darboux problems (1.1), (1.3), and the following conditions

$$
U_{2}\left(x, y_{1}\right)=U_{1}\left(x, y_{1}\right), U_{3}\left(x_{1}, y\right)=U_{1}\left(x_{1}, y\right), U_{s}(x, y)=\left(u_{s, i}(x, y)\right)
$$

take place.

Suppose that

$$
\begin{aligned}
& A_{1}(x, y) \in C(D) \cap C^{(1.0)}\left(D_{1} \cup D_{3}\right), \\
& A_{2}(x, y) \in C(D) \cap C^{(0.1)}\left(D_{1} \cup D_{2}\right),
\end{aligned}
$$

$f[U(x, y)] \in C(\bar{B}), f: \bar{B} \rightarrow \mathbb{R}^{n}, \bar{B} \subset \mathbb{R}^{n+2}$.

It is easy to show that the boundary-value problem (1.1)-(1.3) can be written in the equivalent integral form

$$
\begin{gathered}
U_{s}(x, y)=\Gamma_{S}(x, y)+\epsilon_{s} T_{1, s} F\left[U_{1}(\xi, \eta)\right]+T_{S} F\left[U_{s}(\xi, \eta)\right], \\
(x, y) \in \bar{D}_{s}, s=1,2,3,
\end{gathered}
$$

where

$$
\epsilon_{s}=\left\{\begin{array}{l}
0, s=1, \\
1, s=2,3,
\end{array} \quad F[U(x, y)]:=\left\{\begin{array}{l}
F^{*}[U(x, y)],(x, y) \in D_{1} \cup D_{2}, \\
F^{* *}[U(x, y)],(x, y) \in D_{3},
\end{array}\right.\right.
$$




$$
\begin{gathered}
F^{*}[U(x, y)]:=f[U(x, y)]+\left[A_{2 y}(x, y)+A_{1}(x, y) A_{2}(x, y)\right] U(x, y), \\
F^{* *}[U(x, y)]:=F^{*}[U(x, y)]+\left[A_{1 x}(x, y)-A_{2 y}(x, y)\right] U(x, y), \\
T_{1} F\left[U_{1}(\xi, \eta)\right]:=\int_{x_{0}}^{x} \int_{y_{0}}^{y} K(x, y ; \xi, \eta) F\left[U_{1}(\xi, \eta)\right] d \eta d \xi,(x, y) \in \bar{D}_{1}, \\
T_{2} F\left[U_{2}(\xi, \eta)\right]:=\int_{k_{1}(y)}^{x} \int_{y_{1}}^{y} K(x, y ; \xi, \eta) F\left[U_{2}(\xi, \eta)\right] d \eta d \xi,(x, y) \in \bar{D}_{2}, \\
T_{3} F\left[U_{3}(\xi, \eta)\right]:=\int_{g_{2}(x)}^{y} \int_{x_{1}}^{x} K^{-1}(\xi, \eta ; x, y) F\left[U_{3}(\xi, \eta)\right] d \xi d \eta,(x, y) \in \bar{D}_{3},
\end{gathered}
$$

$K(x, y ; \xi, \eta)=\left(\delta_{i, j} k_{i, j}(x, y ; \xi, \eta)\right), K^{-1}(x, y ; \xi, \eta)=\left(\delta_{i, j} k_{i, j}^{-1}(x, y ; \xi, \eta)\right)$ are some matrixes,

$$
k_{i, i}(x, y ; \xi, \eta):=\exp \left(\int_{x}^{\xi} a_{i, i}^{(2)}(\tau, y) d \tau+\int_{y}^{\eta} a_{i, i}^{(1)}(\xi, \tau) d \tau\right),
$$

$\Gamma_{S}(x, y)=\left(\gamma_{s, i}(x, y)\right), s=1,2,3-$ are vector-functions,

$$
\begin{aligned}
\gamma_{1, i}(x, y):= & \psi_{i}(y) \exp \left(\int_{x}^{x_{0}} a_{i, i}^{(2)}(\xi, y) d \xi\right)+ \\
& +\int_{x_{0}}^{x} k_{i, i}(x, y ; \xi, \eta)\left[\phi_{i}^{\prime}(\xi)+a_{i, i}^{(2)}\left(\xi, y_{0}\right) \phi_{i}(\xi)\right] d \xi,(x, y) \in \bar{D}_{1},
\end{aligned}
$$

Remark 1. If $A_{1 x}(x, y)=A_{2 y}(x, y),(x, y) \in D$ then $F^{*}[U(x, y)] \equiv F^{* *}[U(x, y)]$ and $K(x, y ; \xi, \eta) \equiv K^{-1}(\xi, \eta ; x, y)$. 
According to the problem setting $U_{1 x}\left(x, y_{1}\right)=U_{2 x}\left(x, y_{1}\right)$ and $U_{1 y}\left(x_{1}, y\right)=U_{3 y}\left(x_{1}, y\right)$ when $x \in\left[x_{0}, x_{1}\right], y \in\left[y_{0}, y_{1}\right]$ and

$$
\begin{gathered}
u_{1, i_{y}}\left(x, y_{1}\right)-u_{2, i_{y}}\left(x, y_{1}\right)=\rho_{1, i} \exp \left(\int_{x}^{x_{0}} a_{i, i}^{(2)}\left(\xi, y_{1}\right) d \xi\right), x \in\left[x_{0}, x_{1}\right], \\
u_{1, i_{x}}\left(x_{1}, y\right)-u_{3, i_{x}}\left(x_{1}, y\right)=\rho_{2, i} \exp \left(\int_{y}^{y_{0}} a_{1}\left(x_{1}, \eta\right) d \eta\right), y \in\left[y_{0}, y_{1}\right],
\end{gathered}
$$

where

$$
\begin{gathered}
\rho_{1, i}:=\psi_{i}^{\prime}\left(y_{1}\right)-k_{1}^{\prime}\left(y_{1}\right)\left\{\omega_{i, 1}^{\prime}\left(x_{0}\right)+a_{i, i}^{(2)}\left(x_{0}, y_{1}\right) \omega_{i, 1}\left(x_{0}\right)-\right. \\
-\left[\phi_{i}^{\prime}\left(x_{0}\right)+a_{i, i}^{(2)}\left(x_{0}, y_{0}\right) \phi_{i}\left(x_{0}\right)\right] \exp \left(\int_{y_{1}}^{y_{0}} a_{i, i}^{(1)}\left(x_{0}, \eta\right) d \eta\right)- \\
-\int_{y_{0}}^{y_{1}}\left[f_{i}\left(x_{0}, \eta, \psi_{1}(\eta), \ldots, \psi_{n}(\eta)\right)+\left(a_{i, i \eta}^{(2)}\left(x_{0}, \eta\right)+a_{i, i}^{(1)}\left(x_{0}, \eta\right) a_{i, i}^{(2)}\left(x_{0}, \eta\right) \psi_{i}(\eta)\right)\right] \\
\left.\times \exp \left(\int_{y_{1}}^{y_{0}} a_{i, i}^{(1)}\left(x_{0}, \eta\right) d \eta\right)\right\}, \\
\rho_{2, i}:=\phi_{i}^{\prime}\left(x_{1}\right)-\omega_{i, 2}^{\prime}\left(x_{1}\right)-g_{2}^{\prime}\left(x_{1}\right)\left\{a_{i, i}^{(1)}\left(x_{1}, y_{0}\right) \omega_{i, 2}\left(x_{1}\right)-\right. \\
-\left[\psi_{i}^{\prime}\left(x_{0}\right)+a_{i, i}^{(1)}\left(x_{0}, y_{0}\right) \psi_{i}\left(y_{0}\right)\right] \exp \left(\int_{x_{1}}^{x^{0}} a_{i, i}^{(2)}\left(\xi, y_{0}\right) d \xi\right)- \\
-\int_{x_{0}}^{x_{1}}\left[f_{i}\left(\xi, y_{0}, \phi_{1}(\xi), \ldots, \phi_{n}(\xi)\right)+\left(a_{i, i \xi}^{(1)}\left(\xi, y_{0}\right)+a_{i, i}^{(2)}\left(\xi, y_{0}\right)\right) \phi_{i}(\xi)\right] \\
\left.\times \exp \left(\int_{x_{1}}^{\xi} a_{i, i}^{(2)}\left(\tau, y_{0}\right) d \tau\right) d \xi\right\} .
\end{gathered}
$$

The following lemma holds.

Lemma 1. Let $f[U(x, y)] \in C(\bar{B}), A_{r}(x, y) \in C(D)$,

$r=1,2, A_{1}(x, y) \in C^{(1.0)}\left(D_{1} \cup D_{3}\right), A_{2}(x, y) \in C^{(0.1)}\left(D_{1} \cup D_{2}\right)$ and the boundaryvalue problem (1.1)-(1.3) has the solution.

The solutions of the boundary-value problem (1.1)-(1.3) are regular (it means that $\left.U(x, y) \in C^{*}(\bar{D})\right)$ if and only if the equality

$$
\rho_{r, i}=0,
$$

is true, for all $r=1,2$ and $i=\overline{1, n}$.

In the other case equalities (1.5) hold and the solution of the problem (1.1)-(1.3) is irregular.

Definition 1. We say that the vector-function $F[U(x, y)] \in C_{1}(\bar{B})$ if it satisfies the following conditions [5]:

(1) $F[U(x, y)] \in C(\bar{B})$,

(2) there exists vector-function

$$
H(x, y, U(x, y) ; V(x, y)):=H[U(x, y) ; V(x, y)]
$$


in the space of vector-functions $C\left(\bar{B}_{1}\right), \bar{B}_{1} \subset \mathbb{R}^{2(n+1)}, P r_{x O y} \overline{B_{1}}=\bar{D}$, such that

a) $H[U(x, y) ; V(x, y)] \equiv F[U(x, y)]$,

b) for any pair of vector-functions $U(x, y), V(x, y) \in \bar{B}_{1}$ from the space $C(\bar{D})$ that satisfy condition

$$
U(x, y) \geq V(x, y),(x, y) \in \bar{D},
$$

in the domain $\bar{B}_{1}$, the inequality

$$
H[U(, y) ; V(x, y)]-H[V(x, y) ; U(x, y)] \geq 0
$$

is true,

(3) vector-function $H[U(x, y) ; V(x, y)]$ satisfies the Lipshitz condition in the domain $\bar{B}_{1}$; it means that for any vector-functions $U_{r}(x, y), V_{r}(x, y) \in \bar{B}_{1}$, $r=1,2$ from the space $C(\bar{D})$ the following condition

$$
\left|H\left[U_{1}(x, y) ; U_{2}(x, y)\right]-H\left[V_{1}(x, y) ; V_{2}(x, y)\right]\right| \leq L\left(\left|W_{1}(x, y)\right|+\left|W_{2}(x, y)\right|\right),
$$

holds, where $W_{r}(x, y):=U_{r}(x, y)-V_{r}(x, y), r=1,2, L=\left(\delta_{i, j}, l_{i, j}\right)$ is the Lipshitz matrix, $l_{i, j} \geq 0, i, j=\overline{1, n}$.

If vector-function $f[U(x, y)] \in C(\bar{B})$ and has bounded partial derivatives of the first order on all arguments, starting from the third one, then $F[U(x, y)]$ always belongs to the space $C_{1}(\bar{B})$. The opposite statement isn't true.

Let us set sufficient conditions of existence and uniqueness of regular or irregular solution of the problem (1.1)-(1.3), when $(x, y) \in \bar{D}$.

Suppose that the vector-functions $Z_{s, p}(x, y):=\left(z_{s, i, p}(x, y)\right)$ and $V_{s, p}(x, y):=\left(v_{s, i, p}(x, y)\right) \in C(\bar{D})$ belong to the domain $\bar{B}_{1}, s=1,2,3, p \in \mathbb{N}$.

Let us put

$$
\begin{gathered}
W_{s, p}(x, y)=Z_{s, p}(x, y)-V_{s, p}(x, y),(x, y) \in \bar{D}_{s}, s=1,2,3, \\
f_{s}^{p}(x, y):=H\left[Z_{s, p}(x, y) ; V_{s, p}(x, y)\right], \\
f_{s, p}(x, y):=H\left[V_{s, p}(x, y) ; Z_{s, p}(x, y)\right], \\
\alpha_{s, p}^{*}(x, y):=Z_{s, p}(x, y)-\Gamma_{s}(x, y)-\epsilon_{s} T_{1, s} f_{1}^{p}(\xi, \eta)-T_{s} f_{s}^{p}(\xi, \eta), \\
\beta_{s, p}^{*}(x, y):=V_{s, p}(x, y)-\Gamma_{s}(x, y)-\epsilon_{s} T_{1, s} f_{1, p}(\xi, \eta)-T_{s} f_{s, p}(\xi, \eta), \\
Z_{s, p}^{*}(x, y):=Z_{s, p}(x, y)-C_{s, p}(x, y) W_{s, p}(x, y), \\
V_{s, p}^{*}(x, y):=V_{s, p}(x, y)+Q_{s, p}(x, y) W_{s, p}(x, y), \\
(x, y) \in \bar{D}_{s}, s=1,2,3, p \in \mathbb{N}, \\
F_{s}^{p}(x, y):=H\left[Z_{s, p}^{*}(x, y) ; V_{s, p}^{*}(x, y)\right], \\
F_{s, p}(x, y):=H\left[V_{s, p}^{*}(x, y) ; Z_{s, p+1}(x, y)\right],
\end{gathered}
$$

$C_{s, p}(x, y):=\left(\delta_{i, j} c_{s, i, p}(x, y)\right), Q_{s, p}(x, y):=\left(\delta_{i, j} q_{s, i, p}(x, y)\right)$ are arbitrary matrixes with non-negative elements $c_{s, i, p}(x, y), q_{s, i, p}\left(x, y \in C\left(\bar{D}_{s}\right)\right)$, such that

$$
c_{s, i, p}(x, y) \leq 0,5, q_{s, i, p}(x, y) \leq 0,5,(x, y) \in \bar{D}_{s}, s=1,2,3,
$$


for all $p \in \mathbb{N}$ and $i=\overline{1, n}$.

Let us build the sequences of vector-functions $\left\{Z_{s, p}(x, y)\right\}$ and $\left\{V_{s, p}(x, y)\right\}$ according to the formulas 1.9:

$$
\begin{gathered}
Z_{s, p+1}(x, y)=\Gamma_{s}(x, y)+\epsilon_{s} T_{1, s} F_{1}^{p}(\xi, \eta)+T_{s} F_{s}^{p}(\xi, \eta), \\
V_{s, p+1}(x, y)=\Gamma_{s}(x, y)+\epsilon_{s} T_{1, s} F_{1, p}(\xi, \eta)+T_{s} F_{s}^{p}(\xi, \eta),(x, y) \in \bar{D}_{s},
\end{gathered}
$$

where as the zero approximation $Z_{s, 0}(x, y), V_{s, 0}(x, y) \in \bar{B}_{1}$ we take arbitrary vectorfunctions from $C\left(\bar{D}_{s}\right)$ satisfying conditions (1.2), (1.3) and inequalities

$$
\begin{gathered}
W_{s, 0}(x, y) \geq 0, \alpha_{s, 0}^{*}(x, y) \geq 0, \\
\beta_{s, 0}^{*}(x, y) \leq 0,(x, y) \in \bar{D}_{s}, s=1,2,3 .
\end{gathered}
$$

Afterwards vector-functions $Z_{s, 0}(x, y), V_{s, 0}(x, y) \in C\left(\bar{D}_{s}\right)$ that satisfy conditions (1.2), (1.3), inequalities (1.10) and belong to the domain $\bar{B}_{1}$ will be called comparison functions of the problem (1.1)-(1.3).

From (1.9) follows that for $(x, y) \in \bar{D}_{s}, s=1,2,3$ the equalities

$$
\begin{gathered}
Z_{s, p}(x, y)-Z_{s, p+1}(x, y)=\alpha_{s, p}(x, y):=Z_{s, p}(x, y)-\Gamma_{s}(x, y)- \\
-\epsilon_{s} T_{1, s} F_{1}^{p}(\xi, \eta)-T_{s} F_{s}^{p}(\xi, \eta), \\
V_{s, p}(x, y)-V_{s, p+1}(x, y)=\beta_{s, p}(x, y):=V_{s, p}(x, y)-\Gamma_{s}(x, y)- \\
-\epsilon_{s} T_{1, s} F_{1, p}(\xi, \eta)-T_{s} F_{s, p}(\xi, \eta),
\end{gathered}
$$

are true, where $\alpha_{s, p}(x, y):=\left(\alpha_{s, i, p}(x, y)\right), \beta_{s, p}(x, y):=\left(\beta_{s, i, p}(x, y)\right), i=\overline{1, n}$ are vector-functions and

$$
\begin{gathered}
W_{s, p+1}(x, y)=\epsilon_{s} T_{1, s}\left(F_{1}^{p}(\xi, \eta)-F_{1, p}(\xi, \eta)\right)+T_{s}\left(F_{s}^{p}(\xi, \eta)-F_{s, p}(\xi, \eta)\right), \\
\alpha_{s, p}(x, y)=\epsilon_{s} T_{1, s}\left(F_{1}^{p}(\xi, \eta)-F_{1}^{p+1}(\xi, \eta)\right)+ \\
+T_{s}\left(F_{s}^{p}(\xi, \eta)-F_{s}^{p+1}(\xi, \eta)\right) \\
\beta_{s, p}(x, y):=\epsilon_{s} T_{1, s}\left(F_{1, p}(\xi, \eta)-F_{1, p+1}(\xi, \eta)\right)+ \\
+T_{s}\left(F_{s, p}(\xi, \eta)-F_{s, p+1}(\xi, \eta)\right) .
\end{gathered}
$$

As (1.8), (1.10) hold we get that

$$
V_{s, 0}(x, y) \leq V_{s, 0}^{*}(x, y) \leq Z_{s, 0}^{*}(x, y) \leq Z_{s, 0}(x, y),(x, y) \in \bar{D}_{s}, s=1,2,3 .
$$

It means that

$$
V_{s, 0}^{*}(x, y), Z_{s, 0}^{*}(x, y) \in \bar{B}_{1}
$$

if only $V_{s, 0}(x, y), Z_{s, 0}(x, y) \in \bar{B}_{1}$.

Note that if

then

$$
\alpha_{s, 0}^{*}(x, y) \geq 0
$$

But from (1.11) for $p=0$ we get that

$$
\alpha_{s, 0}(x, y) \geq 0 .
$$

$$
Z_{s, 0}(x, y)-Z_{s, 1}(x, y) \geq 0,
$$




$$
\begin{gathered}
Z_{s, 1}(x, y)-V_{s, 0}(x, y)=-\beta_{s, 0}^{*}(x, y)+\epsilon_{s} T_{1, s}\left(F_{1}^{0}(\xi, \eta)-f_{1,0}(\xi, \eta)\right)+ \\
+T_{s}\left(F_{s}^{0}(\xi, \eta)-f_{s, 0}(\xi, \eta)\right) \geq 0,
\end{gathered}
$$

$Z_{s, 1}(x, y) \in \bar{B}_{1}$ and

$$
\beta_{s, 0}^{*}(x, y)-\beta_{s, 0}(x, y) \geq 0 .
$$

It means that

$$
\beta_{s, 0}(x, y)=V_{s, 0}(x, y)-V_{s, 1}(x, y) \leq 0 .
$$

As $Z_{s, 0}(x, y) \geq V_{s, 1}(x, y)$ then $V_{s, 1}(x, y) \in \bar{B}_{1}$.

We choose matrixes $C_{s, 0}(x, y)$ and $Q_{s, 0}(x, y)$ in such way that for $(x, y) \in \bar{D}_{s}$, $s=1,2,3$, the following conditions

$$
\begin{aligned}
Z_{s, 0}(x, y)-Z_{s, 1}(x, y)-C_{s, 0}(x, y) W_{s, 0}(x, y) & \geq 0, \\
V_{s, 0}(x, y)-V_{s, 1}(x, y)+Q_{s, 0}(x, y) W_{s, 0}(x, y) & \leq 0
\end{aligned}
$$

hold.

It is easy to see that the inequality

$$
\begin{gathered}
V_{s, 0}(x, y)-Z_{s, 1}(x, y)+Q_{s, 0}(x, y) W_{s, 0}(x, y) \leq 0, \\
(x, y) \in \bar{D}_{s}, s=1,2,3,
\end{gathered}
$$

holds if and only if

$$
W_{s, 1}(x, y) \geq 0,(x, y) \in \bar{D}_{s} .
$$

Indeed, if (1.15) holds, from (1.12) for $p=0$, taking into account (1.6), we get that

$$
W_{s, 1}(x, y) \geq 0 .
$$

And if the last estimation is true then from the second inequality (1.14) we establish that condition (1.15) is true.

So

$$
\begin{gathered}
V_{s, 0}(x, y) \leq V_{s, 1}(x, y) \leq Z_{s, 1}(x, y) \leq Z_{s, 0}(x, y), \\
(x, y) \in \bar{D}_{s}, s=1,2,3 .
\end{gathered}
$$

Then from (1.13) for $p=0$ we get

$$
\alpha_{s, 1}(x, y) \geq 0, \beta_{s, 1}(x, y) \leq 0,(x, y) \in \bar{D}_{s}, s=1,2,3 .
$$

Taking vector-functions $Z_{s, 1}(x, y), V_{s, 1}(x, y) \in \bar{B}_{1}$ as the given ones and repeating all the above reasoning by the method of mathematical induction we get that if we choose matrices $C_{s, p}(x, y)$ and $Q_{s, p}(x, y)$ on every step of iterations (1.9) in such way that for $(x, y) \in \bar{D}_{s}, s=1,2,3$ the conditions

$$
\begin{gathered}
Z_{s, p}(x, y)-Z_{s, p+1}(x, y)-C_{s, p}(x, y) W_{s, p}(x, y) \geq 0, \\
V_{s, p}(x, y)-V_{s, p+1}(x, y)+Q_{s, p}(x, y) W_{s, p}(x, y) \leq 0, p \in \mathbb{N},
\end{gathered}
$$


hold, then in the domain $\bar{B}_{1}$ the inequalities

$$
\begin{gathered}
V_{s, p}(x, y) \leq V_{s, p+1}(x, y) \leq Z_{s, p+1}(x, y) \leq Z_{s, p}(x, y), \\
\alpha_{s, p}(x, y) \geq \alpha_{s, p}^{*}(x, y) \geq 0, \beta_{s, p}(x, y) \leq \beta_{s, p}^{*}(x, y) \leq 0, \\
(x, y) \in \bar{D}_{s}, s=1,2,3, \text { forall } p \in \mathbb{N}
\end{gathered}
$$

are true.

Lemma 2. If $F[U(x, y)] \in C_{1}(\bar{B})$ and there exist comparison vector-functions $Z_{s, 0}(x, y), V_{s, 0}(x, y),(x, y) \in \bar{D}_{s}, s=1,2,3$ of the boundary-value problem (1.1)(1.3) in the domain $\bar{B}_{1}$, then the set of matrixes $C_{s, p}(x, y), Q_{s, p}(x, y) \in C\left(\bar{D}_{s}\right)$, that satisfy conditions (1.16), (1.8), in non-empty.

Indeed, let us put

$$
\begin{gathered}
c_{s, i, p}(x, y)=\left\{\begin{array}{l}
\alpha_{s, i, p}^{*}(x, y) \rho_{s, i, p}^{-1}(x, y), W_{s, i, p}(x, y) \neq 0, \\
0, W_{s, i, p}(x, y)=0,
\end{array}\right. \\
q_{s, i, p}(x, y)=\left\{\begin{array}{l}
-\beta_{s, i, p}^{*}(x, y) \rho_{s, i, p}^{-1}(x, y), W_{s, i, p}(x, y) \neq 0, \\
0, W_{s, i, p}(x, y)=0,(x, y) \in \bar{D}_{s}, s=1,2,3,
\end{array}\right. \\
\rho_{s, i, p}(x, y):=\alpha_{s, i, p}^{*}(x, y)-\beta_{s, i, p}^{*}(x, y)+W_{s, i, p}(x, y), p \in \mathbb{N} .
\end{gathered}
$$

It is obvious that the chosen non-negative functions $c_{s, i, p}(x, y), q_{s, i, p}(x, y)$ satisfy conditions (1.8), and according to (1.11)

$$
\begin{gathered}
Z_{s, p}(x, y)-Z s, p+1(x, y)-C_{s, p}(x, y) W_{s, p}(x, y) \geq \\
\geq\left(E-P_{s, p}(x, y)\right) \alpha_{s, p}(x, y) \geq 0 \\
V_{s, p}(x, y)-V_{s, p+1}(x, y)+Q_{s, p}(x, y) W_{s, p}(x, y) \leq \\
\leq\left(E-P_{s, p}(x, y)\right) \beta_{s, p}(x, y) \leq 0 \\
(x, y) \in \bar{D}_{s}, s=1,2,3, p \in \mathbb{N},
\end{gathered}
$$

$P_{s, p}(x, y):=\left(\delta_{i, j}(x, y) W_{s, i, p}(x, y) \rho_{s, i, p}^{-1}(x, y)\right), i, j=\overline{1, n}$ is a matrix.

The following theorem is true.

Theorem 1. Let the vector-function $F\left[U(x, y) \in C_{1}(\bar{B})\right]$, $A_{1}(x, y) \in C(D) \cap C^{(1.0)}\left(D_{1} \cup D_{3}\right), A_{2}(x, y) \in C(D) \cap C^{(0.1)}\left(D_{1} \cup D_{2}\right)$ and there exist the comparison functions $Z_{s, 0}(x, y), V_{s, 0}(x, y) \in \bar{D}_{s}, s=1,2,3$ of the problem (1.1)-(1.3) in the domain $\bar{B}_{1}$.

Then for the vector-functions $Z_{s, p}(x, y), V_{s, p}(x, y)$ built according to (1.9), where non-negative matrixes $C_{s, p}(x, y), Q_{s, p}(x, y) \in C\left(\bar{D}_{s}\right), s=1,2,3$ satisfy conditions (1.8), (1.10) in the domain $\bar{B}_{1}$, the inequalities (1.17) are true.

Let us show that these sequences of vector-functions $\left\{Z_{s, p}(x, y)\right\}$ and $\left\{V_{s, p}(x, y)\right\}$ converge uniformly in the domain $\bar{D}_{s}, s=1,2,3$ to the unique solution of the system of integral equations (1.4). 
As inequalities (1.17) in $\bar{B}_{1}$ hold it is sufficient to show that

$$
\begin{gathered}
\lim _{p \rightarrow \infty} W_{s, p}(x, y)=0, \\
(x, y) \in \bar{D}_{s}, s=1,2,3 .
\end{gathered}
$$

Let

$$
\begin{gathered}
\max _{s, i} \sup _{\bar{D}_{s}}\left|W_{s, i, 0}(x, y)\right|=d, \\
\|L\|=l, \\
\max _{s, i, p} \sup _{\bar{D}_{s}}\left(1-c_{s, i, p}(x, y)-q_{s, i, p}(x, y)\right) \leq q, \\
\max _{s, i} \sup _{\bar{D}_{1} \cup \bar{D}_{s}}\left\{k_{i, i}(x, y ; \xi, \eta), k_{i, i}^{-1}(\xi, \eta ; x, y)\right\} \leq 0,5 K, s=2,3, \\
\max \left\{1, \sup _{\bar{D}}\left(y-y_{0}+x-x_{0}\right)\right\}=\gamma .
\end{gathered}
$$

Then from (1.12) by method of mathematical induction we get that the following estimations

$$
\max _{s, i} \sup _{\bar{D}_{s}} W_{s, i, p}(x, y):=\left\|W_{s, p}(x, y)\right\| \leq \frac{1}{p !}\left(\operatorname{lqk} \gamma n\left(y-y_{0}+x-x_{0}\right)\right)^{p} d,
$$

so

$$
\lim _{p \rightarrow \infty} Z_{s, p}(x, y)=\lim _{p \rightarrow \infty} V_{s, p}(x, y)=U_{s}(x, y)(x, y) \in \bar{D}_{s}, s=1,2,3 .
$$

Passing to the limit in (1.9) for $p \rightarrow \infty$ we get that the limit functions $U_{s}(x, y)$ are the solutions of the systems of integral equations (1.4) for $(x, y) \in \bar{D}_{s}$, $s=1,2,3$.

Theorem 2. Let conditions of Theorem 1 hold.

Then the sequences of vector-functions $\left\{Z_{s, p}(x, y)\right\},\left\{V_{s, p}(x, y)\right\}$ build according to (1.9), where as a zero-approximation we choose the comparison vector-functions of the problem (1.1)-(1.3) and matrixes $C_{s, p}(x, y)$ and $Q_{s, p}(x, y), s=1,2,3$ satisfy conditions (1.8), (1.16):

(1) uniformly converge to the unique solution of the system of integral equations (1.4), for $(x, y) \in \bar{D}_{s}, s=1,2,3$,

(2) estimates (1.18) take place,

(3) in the domain $\bar{B}_{1}$ the inequalities

$$
\begin{gathered}
V_{s, p}(x, y) \leq V_{s, p+1}(x, y) \leq U_{s}(s, y) \leq Z_{s, p+1}(x, y) \leq Z_{s, p}(x, y), \\
(x, y) \in \bar{D}_{s}, s=1,2,3, p \in \mathbb{N},
\end{gathered}
$$

hold, where $U_{s}(x, y)$ is the unique solution of the system of integral equations (1.4), for $(x, y) \in \bar{D}_{s}$,

(4) the convergence of the iteration method (1.9), (1.8), (1.16) isn't slower than the convergence of the Pikar two-sided method (for $C_{s, p}(x, y)=$ $\left.Q_{s, p}(x, y) \equiv 0\right)$. 
Proof. The uniqueness of the solution of the system of integral equations (1.4) for $(x, y) \in \bar{D}_{s}, s=1,2,3$ can be proved from the opposite assumption.

Suppose that for any number $p$ in some point $(x, y) \in \bar{D}_{s} Z_{s, p}(x, y)<U_{s}(x, y)$. Then according to (1.17) for all $m \in \mathbb{N}$

$$
U_{s}(x, y)>Z_{s, p}(x, y) \geq Z_{s, p+m}(x, y)
$$

in the point $(x, y) \in \bar{D}_{s}$ and in this point the sequence of vector-functions $\left\{Z_{s, p+m}(x, y)\right\}$ for $m \rightarrow \infty$ does not converge to the solution $U_{s}(x, y)$. We got the contradiction. Analogically we prove that the inequality

$$
V_{s, p}(x, y) \leq U_{s}(x, y),(x, y) \in \bar{D}_{s}, s=1,2,3
$$

holds.

Let $Z_{s, p}(x, y), V_{s, p}(x, y) \in \bar{B}_{1}$ are the comparison vector-functions of the problem (1.1)-(1.3). Let us put $\bar{Z}_{s, p+1}(x, y), \bar{V}_{s, p+1}(x, y)$ the following two-sided approximations to the solution of the systems of integral equations (1.4) build in order of the Picar method. Then for $(x, y) \in \bar{D}_{s}, s=1,2,3$ according to (1.6), (1.8) we get

$$
\begin{aligned}
& \bar{Z}_{s, p+1}(x, y)-Z_{s, p+1}(x, y)=\epsilon_{s} T_{1, s}\left(f_{1}^{p}(\xi, \eta)-F_{1}^{p}(\xi, \eta)\right)+ \\
& +T_{s}\left(f_{s}^{p}(\xi, \eta)-F_{s}^{p}(\xi, \eta)\right) \geq 0, \bar{V}_{s, p+1}(x, y)-V_{s, p+1}(x, y)= \\
= & \epsilon_{s} T_{1, s}\left(f_{1, p}(\xi, \eta)-F_{1, p}(\xi, \eta)\right)+T_{s}\left(f_{s, p}(\xi, \eta)-F_{s, p}(\xi, \eta)\right) \leq 0 .
\end{aligned}
$$

It means that the convergence of the method is not slower than the convergence of the Picar's two-sided method.

Let us remark that according to the forms of matrices $C_{s, p}(x, y)$ and $Q_{s, p}(x, y)$ in (1.9), (1.8), (1.10) we get different modifications of the two-sided method.

Corollary 1. Let conditions of Theorem 2 hold.

Then there exists the unique solution of the boundary-value problem (1.1)-(1.3) in the domain $\bar{D}$. Besides in the case, when $\rho_{r, i}=0$ for all $r=1,2$ and $i=\overline{1, n}$ it will be regular, and in the other case, irregular.

Corollary 2. Let $\Psi(y)=\Phi(x)=0,(x, y) \in \bar{D}_{1}, \Omega_{r}(x)=0, x \in\left[x_{r-1}, x_{r}\right]$, $r=1,2$ and $F[U(x, y)] \in C_{1}(\bar{B})$, besides $F[U(x, y)] \equiv H[U(x, y) ; 0]$.

Then if $F[0] \leq(\geq) 0$ in $\bar{B}_{1}$ the solution of (1.1)-(1.3) for $(x, y) \in \bar{D}$ satisfies the inequality

$$
U(x, y) \leq(\geq) 0 .
$$

Together with (1.1) let us observe the following system

$$
L_{2} Z(x, y)=f_{1}(x, y, Z(x, y)):=f_{1}[Z(x, y)], f_{1}: \bar{B} \rightarrow \mathbb{R}^{n}, \bar{B} \in \mathbb{R}^{n+2} \text {. }
$$

We suppose that the right hand-sides of the systems (1.1), (1.20) satisfy the following conditions:

(1) $f[U(x, y)] \in C_{1}(\bar{B})$, 
(2) vector-function $f_{1}[Z(x, y)] \in C(\bar{B})$ and has bounded first order derivatives on all its arguments in $\bar{B}$, starting from the third one

$$
\frac{\partial f_{i}[Z(x, y)]}{\partial z_{j}}:=b_{i, j}(x, y)<\infty,
$$

and $\forall(x, y, Z(x, y)) \in \bar{B}$ the inequalities

$$
\begin{aligned}
& b_{i, j}(x, y)+\delta_{i, j}\left[a_{i, j_{y}}^{(2)}(x, y)+a_{i, j}^{(1)}(x, y) a_{i, j}^{(2)}(x, y)\right] \geq 0,(x, y) \in D_{1} \cup D_{2}, \\
& b_{i, j}(x, y)+\delta_{i, j}\left[a_{i, j_{x}}^{(1)}(x, y)+a_{i, j}^{(1)}(x, y) a_{i, j}^{(2)}(x, y)\right] \geq 0,(x, y) \in D_{1} \cup D_{3},
\end{aligned}
$$

hold,

(3) for any vector-function $V(x, y) \in \bar{B}$ from $C^{*}(\bar{D})$

$$
f_{1}[V(x, y)] \geq(\leq) f[V(x, y)] .
$$

Theorem 3. Let $A_{1} \in C(D) \cap C^{(1.0)}\left(D_{1} \cup D_{3}\right), A_{2} \in C(D) \cap C^{(0.1)}\left(D_{1} \cup D_{2}\right)$ and the right sides of the systems (1.1), (1.20) $f[U(x, y)]$ and $f_{1}[Z(x, y)]$ satisfy conditions (1)-(3) above and there exist comparison functions of the problems (1.1)(1.3), (1.20), (1.2), (1.3) in the domain $\bar{B}_{1}$.

Then the solutions of these problems satisfy inequalities

$$
U(x, y) \leq(\geq) Z(x, y),(x, y) \in \bar{D} .
$$

Proof. According to Theorem 2 and Collorary 1 there exist unique solutions of the problems (1.1)-(1.3) and (1.20), (1.2), (1.3) (regular or irregular). So putting $W(x, y):=Z(x, y)-U(x, y)$ and using Lagrange theorem about complete increases we'll get

$$
L_{2} W(x, y)=A_{3}(x, y) W(x, y)+A_{4}(x, y),
$$

where $A_{3}(x, y)=\tilde{b}_{i, j}(x, y), i, j=\overline{1, n}$ matrix, $\tilde{b}_{i, j}(x, y)$ are derivatives of $b_{i, j}(x, y)$ for some fixed values $Z(x, y) \in \bar{B}$ and according to (1.22)

$$
A_{4}(x, y):=f_{1}[U(x, y)]-f[U(x, y)] \geq(\leq) 0 .
$$

Vector-function $W(x, y)$ satisfies homogenous conditions (1.2), (1.3) and

$$
\begin{aligned}
F^{*}[W(x, y)]: & =\left[A_{3}(x, y)+A_{2 y}(x, y)+A_{1}(x, y) A_{2}(x, y)\right] W(x, y)+A_{4}(x, y), \\
F^{* *}[W(x, y)] & :=\left[A_{3}(x, y)+A_{1 x}(x, y)+A_{1}(x, y) A_{2}(x, y)\right] W(x, y)+A_{4}(x, y) \\
& \equiv F^{*}[W(x, y)]+\left[A_{1 x}(x, y)-A_{2 y}(x, y)\right] W(x, y),
\end{aligned}
$$

i.e. according to (1.21)

$$
F[W(x, y)] \equiv H[W(x, y) ; 0]
$$

and

Using Corollary 2 we get that

$$
F[0] \geq(\leq) 0 .
$$

$$
W(x, y) \geq(\leq) 0,(x, y) \in \bar{D} .
$$


It means that inequalities (1.23) hold.

\section{REFERENCES}

[1] L. Bers, F. John, and M. Schechter, Eds., Partial differential equations. Proceeding of the Summer Seminar, Boulder, Colorado, 1957. New York-London-Sydney: Interscience Publishers, a Division of John Wiley and Sons, 1964.

[2] R. Courant, Partial differential equations, New York-London, 1962.

[3] K. Marynets, "On the parametrization of nonlinear boundary value problems with nonlinear boundary conditions," Math. Notes, Miskolc, vol. 12, no. 2, pp. 209-223, 2011.

[4] V. V. Marynets, Analytical methods in the theory of partial differential equations of hyperbolic type. Hoverla, Uzhhorod, 2006.

[5] V. V. Marynets' and A. V. Dobryden', "On a characteristic initial value problem," Nonlinear Oscil., vol. 4, no. 4, pp. 487-499, 2001.

[6] V. V. Marynets and O. Y. Pytovka, "About one boundary-value problem for differential-functional equations of the hyperbolic type," Scientific Transactions of the Uzhhorod University, Series: Mathematics and Informatics, vol. 20, pp. 79-89, 2010.

[7] A. Rontó and M. Rontó, "Periodic successive approximations and interval halving," Math. Notes, Miskolc, vol. 13, no. 2, pp. 459-482, 2012.

[8] M. Rontó and K. Marynets, "On the parametrization of boundary-value problems with three-point non-linear restrictions," Math. Notes, Miskolc, vol. 13, no. 1, pp. 91-106, 2012.

Authors' addresses

V. V. Marynets

Uzhhorod National University, Ukraine

E-mail address: vasyl-marynets@rambler.ru

\section{K. V. Marynets}

Uzhhorod National University, Ukraine

E-mail address: katya_marinets@ukr. net 